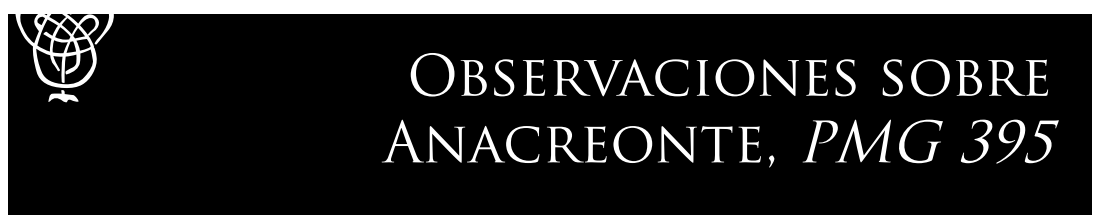

\author{
Alejandro Abritta [Conicet - Universidad de Buenos Aires] \\ [alejandroabritta@gmail.com]
}

Resumen: El propósito del presente artículo es realizar un análisis rítmico-melódico del poema PMG 395 de Anacreonte. Después de una presentación sobre el texto, incluyendo un estudio de las principales discusiones sobre él en la bibliografía, el autor los analiza estudiando la distribución de encabalgamientos y de acentos en las líneas. Se sugiere, finalmente, que las conclusiones obtenidas tienen un alcance que no se limita a $P M G$ 395 en particular, sino que mejoran nuestra compresión de la técnica poética de Anacreonte en sentido amplio.

Palabras clave: Anacreonte - PMG 395 - anacreóntico - métrica griega antigua - música griega antigua

\section{Observations about Anacreon, PMG 395}

Abstract: The goal of this paper is to conduct a rhythmical-melodical analysis of Anacreon's PMG 395. After a presentation of the text, including a study of the main discussions about it in scholarship, the author analyzes it by studying the distribution of enjambments and accents in the lines. Finally, he suggests that the conclusions obtained have a scope that is not limited to $P M G$ 395 in particular, but that they improve our understanding of the poetic technique of Anacreon in general.

Keywords: Anacreon - PMG 395 - Anacreontic - Ancient Greek Metric - Ancient Greek Music

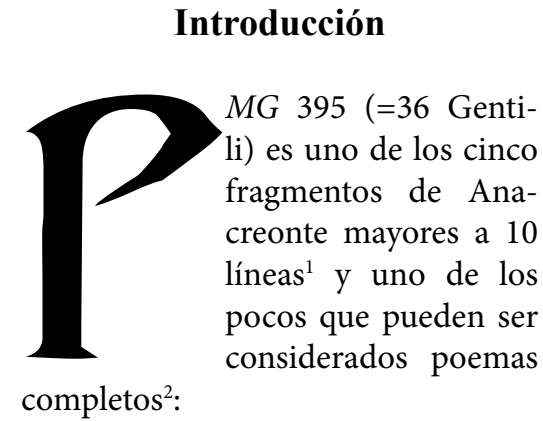

$\pi 0 \lambda$ เoì $\mu \dot{\varepsilon} \nu \eta \dot{\eta} \mu \mathrm{iv} \eta \ddot{\delta} \eta$

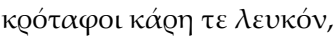

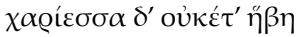

1 Los otros son $346,347,357$ y 388. Para la razón por la que escribo 'líneas' y no 'versos', cfr. n. 11.

2 Cito a partir de la edición de PAge (1962), que de todos modos no difiere de la versión previa de Gentili (1958) más que en la grafía $\alpha$ ó $\alpha \lambda$ én en la 1.10 y el uso de graves en el final de las líneas 5, 9 y 10. Sobre la cuestión de la autenticidad del fragmento, cfr. LAMBIN (2002: 107-111). La traducción es mía y, debo notar, está diseñada con el objetivo de respetar al máximo (aun forzando el español más de la cuenta) la ubicación de las palabras en las líneas. 


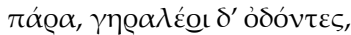
$\gamma \lambda$

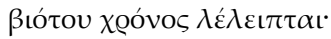

$\delta ı \dot{\alpha} \tau \alpha \tilde{v} \tau^{\prime} \dot{\alpha} v \alpha \sigma \tau \alpha \lambda \hat{v} \zeta \omega$

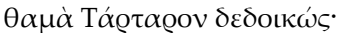

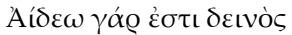
$\mu v \chi o ́ \varsigma, \alpha \grave{Q} \gamma \alpha \lambda \tilde{\eta} \delta^{\prime} \dot{\varepsilon} \varsigma \alpha u ̉ \tau o ̀ v$

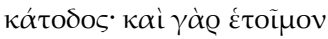

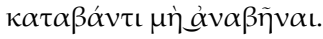

Canosas ahora para mí las sienes y la cabeza, blanca, la graciosa juventud ya no está aquí, los dientes están viejos, y ya no mucho tiempo a la dulce vida le queda;

por esto estoy sollozando a menudo al Tártaro temiendo; pues de Hades es espantoso el abismo y hacia aquel es doloroso 10 el descenso; pues, en efecto, es seguro para el que baja no subirse ${ }^{3}$.

El análisis del texto no ofrece mayores dificultades. Las dos estrofas están perfectamente balanceadas, la primera describiendo la vejez del poeta y la segunda vinculándola a la amenaza del Hades. El único problema significativo de cierto peso es si el cierre del poema tiene un giro humorístico o debemos leer el texto como una expresión seria de preocupación ante la vejez y la muerte ${ }^{4}$. El debate se centra

3 'subirse' busca preservar algo del juego de palabras en la línea final que ha propuesto Giangrande (2011: 31-33) y sobre el que volveré inmediatamente.

4 'Seria' debe entenderse como opuesta a 'humorística', no como opuesta a 'insincera'. No veo razones para dudar de que, si la interpretación de GIANGRANDE es correcta, Anacreonte estaría realmente preocupado en torno al sentido de ḋv $\alpha \beta \alpha$ ív $\omega$ en la 1. 12: para CAMPBELl (1989), entre otros, no tenemos motivos para pensar que significara otra cosa más que 'ascender', es decir, salir del Hades. Sin embargo, en otros contextos ${ }^{5}$, el verbo también se utiliza en el sentido de 'copular', específicamente el acto de 'montar' a una hembra (un tema que encontramos también en $P M G$ 417). La aplicación para humanos está registrada en unos pocos lugares; se encuentra en un fragmento de Aristófanes (344 Kassel-Austin) y en Luciano, Lexiphanes 19, Asinus 51 y 52. La cuestión de fondo es sencilla: ¿podía este sentido del verbo estar suficientemente extendido y fijado como para que el auditorio de Anacreonte entendiera el chiste ${ }^{6}$ ?

ante la imposibilidad de las relaciones carnales en el inframundo.

5 Citados por el mismo Campbell (1989: 49 n. 5). Merece una mención especial CGRN 6 (http://cgrn.ulg.ac.be/file/6/), líneas 6-7,

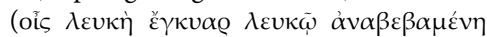
[una oveja blanca preñada, montada por un [carnero] blanco]), porque verifica que el sentido estaba claramente establecido en el s. VI a. C. en Mileto, vecina de Teos, patria de Anacreonte, y de la corte de su patrón más importante, Polícrates de Samos.

6 Debe señalarse un punto que, si bien no suma al argumento, es importante no ignorar: un simple gesto del recitador bastaría para resolver la ambigüedad incluso si el sentido sexual de $\alpha \dot{v} \alpha \beta \alpha \alpha^{\prime} v \omega$ no hubiera estado del todo extendido. Es de común conocimiento que pocas palabras son incapaces de tener un sentido obsceno en el contexto y con la, si se me permite, performance adecuada. No podemos de ningún modo asumir que este era el caso en $P M G$ 395 , pero la posibilidad de que lo fuera no puede escapársenos tampoco. 
No es posible resolver este problema; sin embargo, la alternativa humorística permite una interpretación del poema coherente internamente y con el resto de la obra de Anacreonte:

The structure is very neat (...): the final $\alpha v \alpha \beta \tilde{\eta} v \alpha \iota$ ("copulate") explains the preceding $\gamma \lambda u \kappa \varepsilon \rho o v$ and $\pi 0 \lambda \lambda$ ó 5 , which remain meaningless until we reach the final point. For what reason can Anacreon call his present exist-

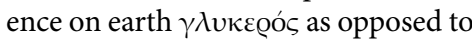
the existence which he will continue in Hades? The reason is finally revealed

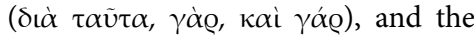
penny thus neatly drops: it was a topos (cfr. e.g. A.P. VII 25,5-8 on this topos applied to Anacreon!) that in Hades one could not copulate: $\alpha v \alpha \beta \tilde{\eta} v \alpha$ เ means "copulate", in Anacreon's poem: the poet is old, but still gifted with amatory powers (hence his $\gamma \lambda \nu \kappa \varepsilon-$ @oũ), and only a very short space of such $\gamma \lambda$ ккеó́ (amatory) life is left to him, now that he is old, because when he is in Hades he will no longer be able to copulate. In other words: the word $\alpha v \alpha \beta \tilde{\eta} v \alpha$, which Anacreon has, with felicitous ambiguity, placed at the end of his poem, cannot, in its context, mean "go up from Hades", as the reader is at first misled to believe by the cleverly contrived opposition $\kappa \alpha-$ $\tau \alpha \beta \alpha \dot{\alpha} v \tau \mathrm{L} / \dot{\alpha} v \alpha \beta \tilde{\eta} v \alpha \mathrm{s}$, because the fact that one cannot go up to earth from Hades is irrelevant to the difference between existence on earth and existence in Hades, which difference is the

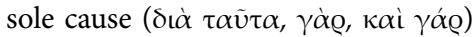
of Anacreon's worry. To Anacreon,

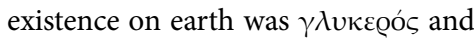
existence in Hades was $\delta \varepsilon เ v o ́ \zeta$ because on earth one could copulate, whereas in Hades one could not. Existence in Hades was not bad in itself (cfr. the commentators on Callim. fr. 191,1 f. Pf.): Anacreon would not regret continuing his existence there, were it not for one thing, i.e. the impossibility to make love ${ }^{7}$.

Si bien se podría objetar la idea de que el Hades no es motivo de temor en sí mismo ${ }^{8}$, el juego de palabras no necesita rechazar ese miedo: el humor de la situación parecería estar en que la amenaza de la muerte resulta menos terrible en sí misma que porque implica el final de la existencia erótica, que, debe inferirse, no le ha llegado todavía a este hombre canoso y con dientes viejos?.

Desde el punto de vista métrico, el poema no ofrece dificultades. Las estrofas están compuestas por cuatro 'anacreónticos' $\left(\mathrm{kkl} \mathrm{kl} \mathrm{kl} \mathrm{a}=\right.$ polss $\left.{ }^{10}\right)$,

7 Giangrande (1973: 131 n. 5). Cfr. también GIANGRANDE (1968: 109-111 y 2011: 31-33).

8 La interpretación de GiANGRANDE es por lo menos simplista: la vida de ultratumba entre los griegos no puede interpretarse como una mera continuidad de la terrena. Cfr. e.g. Pín., Ol. 2.56-80, con la interpretación de Torres (2007: 284-360).

9 Una lectura inevitable en el contexto de la poesía de Anacreonte; cfr. Kantzios (2010).

10 Utilizo el sistema de notación inaugurado por Dale (1969: 41-97), que he explicado y defendido Abritta (en prensa). En pocas palabras: $d=1 \mathrm{kkl}, \mathrm{s}=1 \mathrm{kl}, \mathrm{dd} / \mathrm{ss}$ [prolongación] $=$ I kkl kkl $/ \mathrm{kl} \mathrm{kl}$, d.d/ sls [yuxtaposición] = I kkl I kkl / l kl I kl . El signo de anceps $(\mathrm{X})$ significa lo mismo que en cualquier otra notación, pero debe observarse que también pueden ser ancipitia en un nivel abstracto elementos que se realizan siempre como una sílaba larga. Los versos pueden tener final 'sordo', cuando el cierre coincide con el final de una unidad (e.g. XXds; señalo, en el esquema desagregado, el carácter variable de la sílaba final con 
seguidos de un remate en dos versos: una secuencia $\mathrm{kkl}$ I kkl a (= polídx) y un último anacreóntico ${ }^{11}$. La intención rítmica es fácil de inferir: después de una serie de repeticiones del ritmo del poema que parecerían funcionar a modo de 'amague' estíquico, el cambio hacia polídx traiciona las expectativas del oyente en el punto clave donde se espera la repetición $\mathrm{kl} \mathrm{Kl}$ (las dos secuencias componentes comienzan rel... $\left.{ }^{12}\right)$. Después de esto, la repetición del anacreóntico en el cierre de la estrofa (donde es dable inferir una pausa, sugerida además por la sintaxis) ofrecería un, por así decirlo, alivio rítmico a los sorprendidos receptores.

Mi propósito en lo que sigue es mostrar la técnica compositiva a través de la cual ambos aspectos del poema

U en estos casos -i.e. $x x \mathrm{l} \mathrm{kkl} \mathrm{ku)} \mathrm{o} \mathrm{'sonoro',}$ cuando el cierre está un elemento después del final de la última unidad (e.g. Xxdsa; nótese el uso de a para distinguir este final variable del sordo). En el comienzo de los versos, el primer elemento de una unidad puede omitirse ('acefalía', señalada con $p$, como en pelssx.

11 La estructura de la estrofa se analiza pelssx| pelssx| pelssx| polssx| polídx| pelssal. Nótese que no hay división estricta (indicada por $\mathrm{U}$ entre las unidades mayores (es decir, pelssx y polídx), sobre las que no es posible asegurar si son cola o versos individuales (de ahí el uso de $\mathrm{X}$ en lugar de $\mathrm{a}$ al final de cada uno, excepto el último). Más sobre esto abajo. Sobre la métrica de Anacreonte en general, cfr. SiCKING (1993: 156-159); el análisis que ofrece WEST (1982: 56-59) es un estudio de casos sin pretensión aparente de lograr una comprensión integral de la técnica poética.

12 Las palabras ubicadas en la sílaba larga inesperada no parecen producto de la casualidad: oủкর́cı en la línea 5 ("NO mucho de

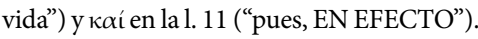

trabajan en conjunto. La aparente simplicidad rítmica esconde una manipulación compleja de las expectativas métrico-rítmicas que, a pesar de estar en una composición de apenas 12 líneas (y menos de 50 palabras), muestran que la técnica poética de Anacreonte contaba con recursos de cierta sofisticación. Me concentraré en dos rasgos que trabajan en paralelo: el uso de encabalgamientos y la distribución de acentos en puntos clave del poema.

\section{Análisis rítmico-acentual}

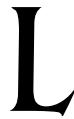

a utilización del recurso del encabalgamiento salta a la vista, pero no ha sido tomada en cuenta por los críticos: se encuentra entre las líneas 1 y 2, 3 y 4, 5 y 6, 7 y 8 , $9,10,11$ y 12 . La distribución es elegante, como puede verse, y podría sugerir una interpretación de la estrofa de tipo pelssx| pelssuU pelssx| relssuU polídx| polssul (es decir, en dísticos). Nótese, sin embargo, que hacia el final del poema esta organización es traicionada. En efecto, aunque el encabalgamiento fuerte en la 1.9 no es del todo inesperado tomando en cuenta lo precedente, el de la l. 10 sí lo es y unifica las últimas cuatro líneas como una única gran secuencia. La segunda estrofa, así, presenta una estructura diferente a la primera: $\mathrm{pdss \textrm {x }}$ pelssuU polssx| pelssx| pelídx| pelssul.

Existe otra diferencia entre las dos partes del poema: mientras que los tres encabalgamientos de la primera estrofa son fuertes, el que une los vv. 7 y 8 es particularmente débil, al punto que el adverbio $\theta \alpha \mu \alpha$ podría interpre- 
tarse como subordinado al participio que le sigue. La larga secuencia final, así, está precedida por un refuerzo de las unidades componentes del poema.

Otro rasgo que separa la última parte del texto del resto es la distribución de tonos. Aunque ha habido mucho debate sobre la relación entre la música griega antigua y el contorno tonal de las palabras, es bastante estándar en lenguajes con acento tonal que exista una coordinación entre el tono acentual y el musical, como han observado Devine y Stephens (1994: 162-171). Esta relación se modifica en la historia de la poesía griega (en particular, con la aparición del fenómeno de la 'Nueva Música'), pero, como ha demostrado Сомотті (1989) a partir del análisis del testimonio antiguo y los importantes resultados de WAHLSTRÖM (1970), la hipótesis más plausible es que, en el periodo arcaico y hasta finales del siglo $\mathrm{V}$ los tonos acentuales eran respetados, al menos hasta cierto punto, en la composición de las melodías musicales ${ }^{13}$. Esto no implica que podamos reconstruir las melodías simplemente a partir de los acentos, pero sí sugiere que, estudiando su distribución, es posible ubicar los movimientos tonales ascendentes y descendentes.

Para ello es necesario apelar a la teoría sobre el acento griego desarrollada por Allen (1973: 230-255) y Devine y Stephens (1994: 171-194),

13 El problema ha sido discutido también por Ruijgh (2001: 303-315) e Irigoin (2006: 224-226), sin que estos debates hayan aportado demasiado a las conclusiones alcanzadas por Сомотті. de la cual deben destacarse cuatro puntos clave:

1. El acento griego antiguo consistía (como, por ejemplo, el del japonés) en un contorno tonal -es decir, un ascenso del tono seguido de un descenso-, no solo del ascenso del tono que caracteriza al acento de lenguajes como el español y el inglés.

2. Los agudos y circunflejos señalan el ascenso del tono (circunflejo significa "ascenso del tono en la primera sílaba de una vocal larga").

3. Cuando hay una sílaba larga después de un acento agudo, el tono llega por debajo del nivel tonal de las sílabas no-acentuadas, produciendo un contorno medio-alto-bajo característico. Como he intentado demostrar en ABRITTA (2015), la coincidencia de sílaba larga y descenso del tono era de particular importancia en la composición poética.

4. Aunque ha habido mucho debate sobre la naturaleza del grave ${ }^{14}$, la evidencia musical sugiere que era un agudo debilitado, indicando un ascenso del tono de alrededor de un tercio del de aquel.

Utilizando estos principios es posible construir un 'mapa melódico' de un poema lírico, señalando los puntos de ascenso y descenso del tono. Aunque no podemos estar seguros del grado de esos movimientos, tene-

14 Cfr. Devine y Stephens (1994: 180-183), DaneK y Hagel (1995: 5). 
mos una indicación clara de su dirección, si la melodía seguía o respetaba el contorno tonal de las palabras.

El siguiente 'mapa' muestra los ascensos y descensos del tono en $P M G$ 395. 'A/a' señala 'ascenso del tono, ' $D$ ', 'descenso del tono'. Por supuesto, no pretendo sugerir que el tono estuviera subiendo constantemente con secuencias de ascensos, sino que en cada uno sube respecto al tono medio, la nota que denominaríamos 'tónica'. La mayúscula indica que el movimiento se da en una sílaba larga, la minúscula en una breve; utilizo cursiva para marcar los graves y los signos habituales para largas y breves para las sílabas no acentuadas.
La organización melódica del poema destaca dos puntos con sucesiones descendentes: la l. 6 y la l. 9. El primero es un punto natural para estos declives, no solo porque está al final de la primera secuencia y el descenso del tono refuerza el cierre ${ }^{18}$, sino también porque esta caída marcada podría enfatizar la queja sobre el final de la vida ${ }^{19}$.

La segunda estrofa inicia con un fuerte contraste rítmico: las líneas 7 y 8 empiezan de forma semejante (grave seguido de ascenso fuerte), pero terminan en direcciones opuestas. La oposición melódica entre $\dot{\alpha} v \alpha \sigma \tau \alpha$ $\lambda \dot{v} \zeta \omega$ y $\delta \varepsilon \delta o \iota \kappa \omega ́ \varsigma$, anuncia el cambio que se da en la 1. 9, donde, después del

\begin{abstract}
$\pi$

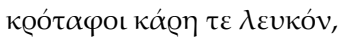

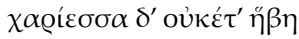

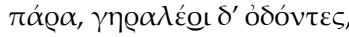

$\gamma \lambda$

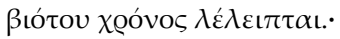

$\delta \grave{\alpha} \tau \alpha \tilde{v} \tau^{\prime} \dot{\alpha} v \alpha \sigma \tau \alpha \lambda \hat{\zeta} \zeta \omega$

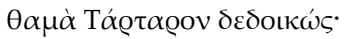

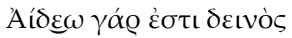

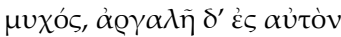

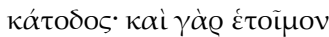

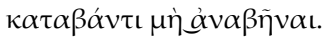

$\mathrm{kk} A a \mathrm{l} a \mathrm{AD}$

akl aDkl A

$\mathrm{kaDkl}$ aAD

akl kA ${ }^{15} k A k$

kkAl akl $a$

$\mathrm{kaDaDaDk}$

kaAkl kAD

kaAkl kl A

$\mathrm{kaDaD}^{16} \mathrm{kl} a$

kal kAkl $a$

akl AakAk

kkAk $A^{17} \mathrm{kAk}$
15 La contracción de ćoı funciona prosódicamente como un circunflejo, es decir, como una sílaba larga con ascenso del tono en la primera mora.

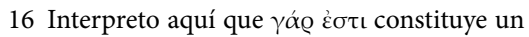
grupo apositivo, es decir una única palabra métrica, por lo que el contorno acentual puede atravesar el límite entre ambas.

17 Asumo aquí que la contracción entre $\mu \grave{y}$ y $\alpha \dot{\alpha} \alpha \beta \tilde{\eta} v \alpha$ เ funciona como una prodelisión, porque el grave resultaría importante para que el oyente reconociera la partícula negativa. En cualquier caso, no modifica el análisis.
18 Devine y Stephens (1994: 429-431).

19 Recuérdese que el término $\beta \alpha \varrho v ́ c$, utilizado para los tonos bajos, también quiere decir 'pesado', 'gravoso'. Uno se sentiría tentado de asociar también el descenso del tono a la 'caída' al Hades, pero "The Greeks did not as a rule speak of 'high' and 'low' pitch." (West, 1992: 64 n. 73). Hay algunas excepciones (cfr. Ps. Arist., Pr. 19.3.37.47 y BARKER, 1989, 384-385), pero son muy posteriores a Anacreonte y parecen atravesadas por concepciones teóricas que este difícilmente manejara. 


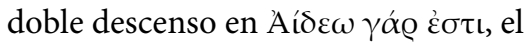
tono no vuelve a bajar en lo que queda del poema.

Es difícil analizar esto sin un modelo para la interpretación de las técnicas melódicas en la lírica, del que carecemos. En un trabajo anterior, he observado que un cambio de tono semejante (de orden inverso), marca en Íbico, $P M G 286$ el paso de un locus amoenus (en tonos altos) a una tormenta (en tonos bajos y luego mezclados). Si el mismo esquema se repite aquí, sin caer en el reduccionismo de asociar tonos altos con 'alegría y bienestar' y tonos bajos con 'sufrimiento y dolor', sí parecería que, donde se esperaría la repetición del cierre de la primera estrofa con su queja por el final de la vida, Anacreonte sorprende con una sucesión ininterrumpida de ascensos. Por otro lado, es necesario notar que, dado que los tonos descendentes caracterizan y marcan el final de las secuencias, su evasión sistemática en un pasaje podría implicar también una aceleración del ritmo, coherente, en este caso, con la acumulación de encabalgamientos.

Este trabajo conjunto de los recursos rítmicos y acentuales se observa en otros lugares. Nótese la re-

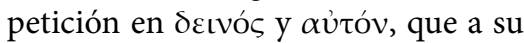
vez repite la del encabalgamiento más fuerte de la primera estrofa en el final

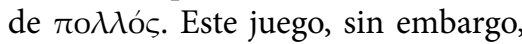
ya se da en los primero cuatro versos:

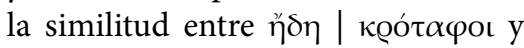
$\eta \eta \beta \eta \mid \pi \alpha ́ @ \alpha$ salta a la vista; nótese en particular la rima entre las líneas 1 y 3. Merece señalarse también, en este sentido, la coincidencia de que en las líneas 2 y 4 se anticipan los cierres de los últimos cuatro versos, en $\lambda \varepsilon v \kappa o ́ v$

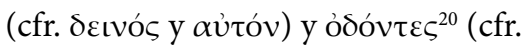

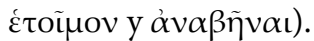

Es posible especular sobre un último punto. El poema se cierra con tres palabras (descontando el contraído $\mu$ ì) prosódicamente properis-

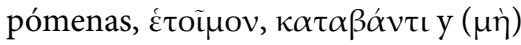
$\dot{\alpha} v \alpha \beta \tilde{\eta} v \alpha$ t. Si se recuerda que uno de los nombres griegos para el circunflejo era ò $\hat{v} \beta \alpha \varrho ı \varsigma^{21}$, esta sucesión podría estar jugando con el sentido del

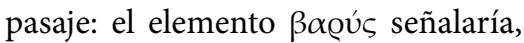
así, el peso (¿de la tierra?) que, en el Hades, hace imposible 'montar' a los que han caído ${ }^{22}$.

20 Sobre la interpretación de estas formas de final trocaico con sílaba cerrada por líquida $\mathrm{y}$ acento agudo en penúltima como prosódicamente perispómenas, cfr. ABRITTA (2017: 72-75, con sus referencias).

21 Cfr. Jacoвy 2a, 70, Fr. 108 (prosodieen sind $\beta \alpha \varrho \varepsilon \tilde{i} \alpha$, ò $\xi \varepsilon \tilde{\imath} \alpha, \mu \varepsilon \dot{\sigma} \eta$. quartae illi [coniungit], quia ceteris perplexior est, plura sunt vocabula: Ammonius Alexandrius, qui Aristarchi scholae successit, ò Ł́́ $\beta \alpha \varrho v v$ vocat). También las descripciones en D. H., Comp. Verb. 40.17-42.14 y Arcadio 186.16-188.11 que, si bien no utilizan el neologismo, explican al circunflejo ( $\pi \varepsilon \rho \iota \sigma \pi \omega ́ \mu \varepsilon v o v)$ como la combinación de ỏçús y $\beta \alpha \varrho u ́ s$.

22 Una hipótesis alternativa puede mencionarse: dado que el tono 'alto' en griego antiguo a veces se asocia a lo que nosotros llamamos tono bajo (puesto que las cuerdas de la lira iban de grave a agudo, empezando desde arriba), quizás la no aparición de tonos bajos en la última parte está expresando metafóricamente la imposibilidad de subir. Es difícil saber si esto es un prejuicio cultural, pero la hipótesis no me resulta convincente. 


\section{Conclusiones}

$P$ $M G$ 395, como puede verse, es un poema cuidadosamente elaborado para marcar las unidades rítmicas y semánticas. La primera estrofa se organiza en dísticos, los primeros dos de los cuales están asociados por la rima de sus componentes iniciales. El cierre de esta estrofa está marcado por la única sucesión del poema de tres palabras con tono descendente. Las líneas 7 y 8 parecieran funcionar como transición: aunque componen un dístico unido por un ligero encabalgamiento, sus comienzos melódicamente similares y las palabras finales terminadas en omega las demarcan como unidades paralelas y, al menos en parte, independientes. En la línea que sigue comienza a precipitarse el cierre, primero con la interrupción de los tonos descendentes después de $\gamma \alpha \dot{\varrho} \varrho \dot{\varepsilon} \sigma \tau \iota$ y luego con la sucesión de encabalgamientos que comienza en $\delta \varepsilon เ v o ́ \varsigma$. Estas últimas cuatro unidades, en efecto, parecen anticipar el remate del poema (que, nótese, está compuesto solo por la última palabra) con una aceleración y precipitación del ritmo y la melodía.

Estas observaciones permiten una comprensión más cabal de la técnica que Anacreonte ha utilizado en $P M G$ 395, pero también refuerzan la afirmación final de Sicking (1993: 159) sobre la poesía del autor:

Beispiele wie diese verstärken die Unsicherheit bezüglich der Frage, ob wir mit Hilfe des üblichen Unterschieds zwischen Vers und Strophe der eigenartigen Kompositionsweise
Anakreons -dessen Verse in ihren Verhältnis zum Ganzen öfter wie die Kola eines Verses wirken- gerecht werden können.

Esta ambigüedad en la naturaleza de las unidades compositivas de Anacreonte, que parecen estar a mitad de camino entre versos de pleno derecho y cola componentes de verso, explica cómo le era posible trabajar al mismo tiempo con encabalgamientos tan fuertes como los que se han observado en $P M G 395$ y, a la vez, con un recurso como la rima entre las unidades, que demanda que estas estén delimitadas de forma clara. La lección que el presente análisis parecería dejar, por ello, es que nuestra comprensión del ritmo de la poesía griega antigua puede mejorar si expandimos algunos de nuestros conceptos para adecuarlos a las técnicas de los autores, en lugar de adaptar las técnicas de los autores a nuestros conceptos restringidos.

\section{Ediciones y traducciones}

BArker, A. (1989). Greek Musical Writings. Vol. II: Harmonic and Acoustic Theory. Cambridge: Cambridge University Press.

Gentili, B. (1958). Anacreonte. Roma: Edizioni dell'Ateneo.

Page, D. L. (1962). Poetae Melici Graeci = $P M G$. Oxford: Clarendon Press.

\section{Bibliografía citada}

Abritta, A. (2015). "On the Role of Accent in Ancient Greek Poetry. Pitch Patterns in the Homeric Hexameter". 
En Quaderni Urbinati di Cultura Classica 111; 11-27.

Abritta, A. (2017). Hacia una historia coral de los metros griegos: Rasgos formales de los metros no-líricos desde la época arcaica hasta la antigüedad tardía. Tesis de Doctorado, Buenos Aires: Universidad de Buenos Aires, Facultad de Filosofía y Letras. Disponible en http://repositorio.filo.uba.ar:8080/ xmlui/handle/filodigital/3335.

Abritta, A. (en prensa). "Consideraciones preliminares para un nuevo modelo teórico de la métrica griega antigua: el caso del verso eólico". Synthesis 25 .

Allen, W. S. (1973). Accent and Rhythm. Cambridge: Cambridge University Press.

Сомотті, G. (1989). “Melodia e accento di parola nelle testimonianze degli antichi e nei testi con notazione musicale". En Quaderni Urbinati di Cultura Classica 32; 91-108.

Dale, A. M. (1969). Collected Papers. Cambridge: Cambridge University Press.

Danek, G. y Hagel, S. (1995). "Homer-singen". En Wiener Humanistische Blätter 37; 5-20.

Devine, A.M. y Stephens, L. D. (1994). The Prosody of Greek Speech. New York: Oxford University Press.

Giangrande, G. (1968). "Sympotic Literature and Epigram", en Raubitschek, A. E. et al. L'Épigramme Grecque. Genève: Fondation Hardt; 91-174.

Giangrande, G. (1973). "Anacreon and the Lesbian Girl". En Quaderni Urbinati di Cultura Classica 16; 129-133.

Giangrande, G. (2011). “Anacreon's Sense of Humour and the Greek Language". En Habis 42; 27-33.

Irigoin, J. (2006). "Euripide poète et musicien selon Denys d'Halicarnasse". En Pallas 72; 219-227.
Kantzios, I. (2010). "Marginal Voice and Erotic Discourse in Anacreon". En Mnemosyne 63; 577-589.

Lambin, G. (2002). Anacréon. Fragments et imitations. Rennes: Presses Universitaires de Rennes.

Ruijgh, C. J. (2001). "Le 'Spectacle des lettres', comédie de Callias (Athénée $X$ 453c-455b)., avec un excursus sur les rapports entre la mélodie du chant et les contours mélodiques du langage parlé”. En Mnemosyne 54; 257-335.

Sicking, C. M. J. (1993). Griechische Verslehre. München: C. H. Beck.

Torres, D. A. (2007). La escatología en la lírica de Píndaro y sus fuentes. Buenos Aires: Facultad de Filosofía y Letras de la Universidad de Buenos Aires.

Wahlström, E. (1970) Accentual Responsion in Greek Strophic Poetry, Helsinki: Finnish Academy of Science.

West, M. L. (1982). Greek Metre. Oxford: Oxford University Press.

West, M. L. (1992). Ancient Greek Music. Oxford: Oxford University Press.

Recibido: 11-09-2018

Evaluado: 21-09-2018

Aceptado: 22-09-2018 